\title{
NATURAL HISTORY NOTE \\ Nuevos registros de murciélagos en la pequeña y remota isla de Alborán (Almería, Spain, Mediterráneo occidental)
}

\author{
Mariano Paracuellos ${ }^{1}$, Jorge Tortolero², Elena Migens ${ }^{1}$, Juan C. Nevado ${ }^{3}$
}

\begin{abstract}
${ }^{1}$ Gestión de la Biodiversidad y Geodiversidad (Agencia de Medio Ambiente y Agua, Consejería de Agricultura, Ganadería, Pesca y Desarrollo Sostenible, Junta de Andalucía), Cl. California 2 pl. baja, 04007, Almería, Spain
\end{abstract}

${ }^{2}$ Destacamento Naval de Alborán (Fuerza de Acción Marítima en Cádiz, Ministerio de Defensa), Almería, Spain

${ }^{3}$ Dpto. de Geodiversidad y Biodiversidad (Consejería de Agricultura, Ganadería, Pesca y Desarrollo Sostenible, Junta de Andalucía), Cl. Hermanos Machado 4 4a pl., 04004, Almería, Spain

*Corresponding author: mariano.paracuellos@juntadeandalucia. es

DOI: https://doi.org/10.14709/

BarbJ.13.1.2020.06

Palabras clave: Mar de Alborán, migración, movimientos intercontinentales, quirópteros.

Keywords: Alborán sea, intercontinenta movements, migration, chiroptera.

received: November, 5th 2019 accepted: April, 22nd 2020

\begin{abstract}
RESUMEN
Un buen conocimiento sobre los posibles lugares de paso migratorio de quirópteros es esencial para entender sus patrones de desplazamiento estacional y adaptar las medidas de conservación que los incumben. En la presente nota actualizamos la información acerca de la presencia de murciélagos detectados en la isla de Alborán, un remoto enclave del Mediterráneo occidental entre África y Europa. Aunque existen varias observaciones aisladas de quirópteros en la isla, hasta la fecha tan solo se había publicado la identificación de un registro puntual de Tadarida teniotis. En esta aportación se amplía la información de la presencia de este grupo en primavera y otoño, incluyendo otro registro de la misma especie y la primera cita conocida de Hypsugo savii en el lugar. Estos hallazgos se relacionan con ejemplares esporádicos de murciélagos observados en la isla que volaban, probablemente, entre África y Europa. Los datos sugieren la posibilidad de desplazamientos intercontinentales de quirópteros sobre el mar entre sus orillas africanas y europeas.
\end{abstract}

\begin{abstract}
Good knowledge about the possible stopovers of migratory passages of chiroptera is essential to understand their patterns of seasonal displacement and adapt the conservational measures involved. In this article, information is actualized on the presence of bats detected on the Alborán island, a remote enclave of the western Mediterranean between Africa and Europe. Although there are several isolated observations of chiroptera on the island, to date only one record identified of Tadarida teniotis has been published. This contribution expands the information of this group in spring and autumn, including another record of the same species and the first known cite of Hypsugo savii at the island. These findings are related to sporadic specimens of bats observed at the island flying, probably, between Africa and Europe. The data suggests the possibility of intercontinental movements of chiroptera over the sea between their African and European shores.
\end{abstract}

El estudio de los movimientos de dispersión y migratorios de los quirópteros ha adquirido recientemente un nuevo interés (Hutterer et al. 2005, Amengual et al. 2007, Boshamer \& Bekker 2008, Ahlén et al. 2009, Bisson et al. 2009, Hedenström 2009, Popa-Lisseanu \& Voigt 2009, Rydell et al. 2014, Hüppop \& Hill 2016). Por eso, cualquier dato relativo a lugares y rutas que tales animales puedan estar utilizando para sus desplazamientos es de gran interés y muy relevante para su conservación (Barova \& Streit 2018).

En este contexto, resulta importante conocer datos acerca de la posible conectividad entre las poblaciones de las especies de murciélagos presentes en el Magreb y la península Ibérica, separadas por el mar de Alborán donde dominan las aguas abiertas (García-Mudarra et al. 2009), con tan solo una solitaria, reducida y remota isla que le da nombre en su centro. En este extremo del Mediterráneo, además, la información acerca de los murciélagos en sus islas es casi testimonial (Ibáñez 2007, Yus et al. 2013, Jiménez-Robles et al. 2018). Dada la relevancia de este tipo de registros, en esta nota se actualiza la información acerca de la presencia de murciélagos en el remoto enclave marítimo de la isla de Alborán, donde hasta la fecha solo se tenían avistamientos ocasionales y una cita puntual de Tadarida teniotis (Rafinesque, 1814).

Aún a pesar de su reducido tamaño $(7,12$ ha) y distante localización (a $55,5 \mathrm{~km}$ al norte de las costas africanas y a $85 \mathrm{~km}$ al sur de las ibéricas) en el extremo oeste del mar Mediterráneo $\left(35^{\circ} 56^{\prime} 22^{\prime \prime} \mathrm{N} \quad 3^{\circ} 2^{\prime} 67^{\prime \prime} \mathrm{W}\right.$, Fig. 1, Almería, España), la isla de Alborán presenta una gran importancia para la conservación de la biodiversidad, por lo que se ha 


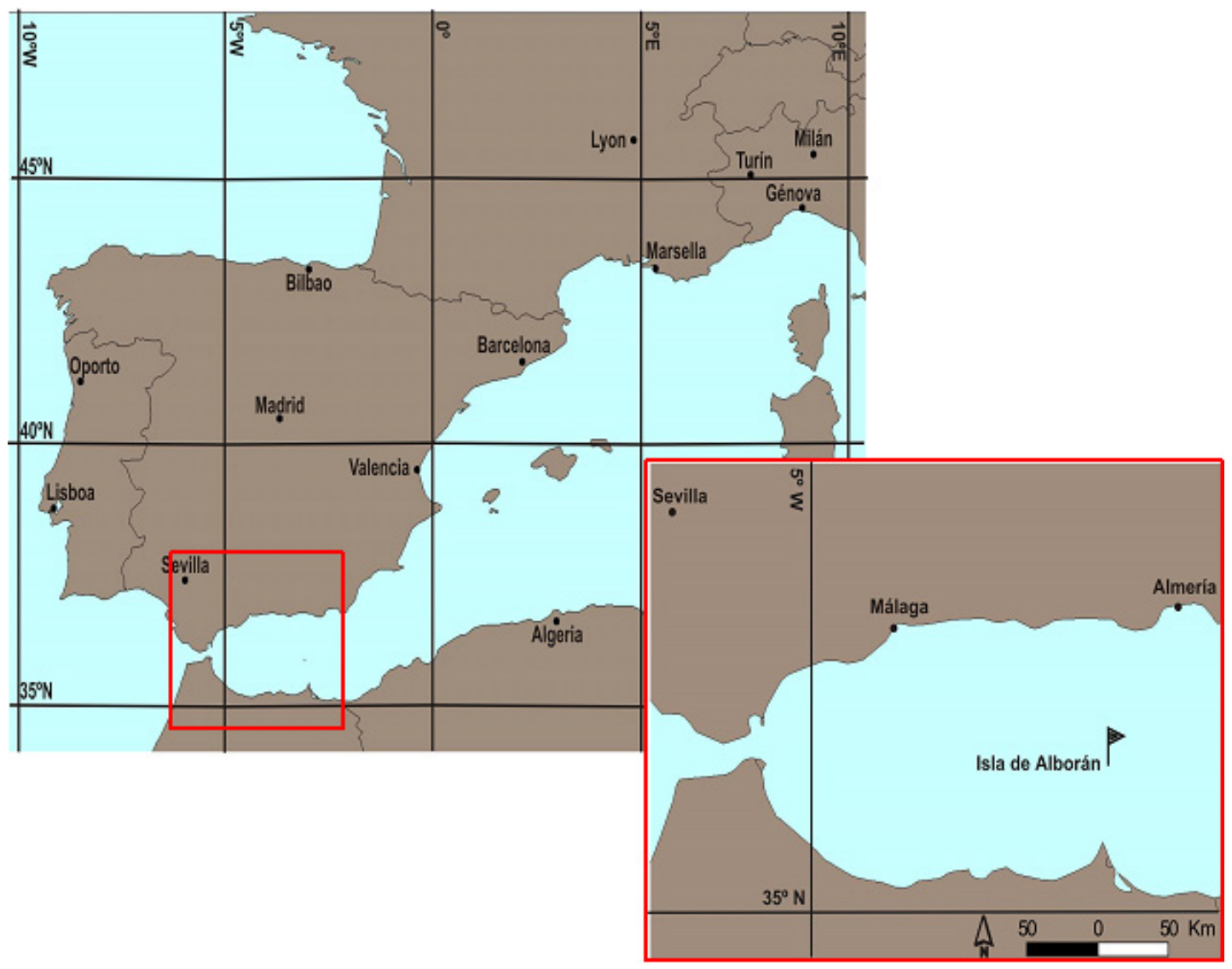

Fig. 1 - Localización geográfica de la isla de Alborán (mapa extraído de Paracuellos et al. 2006).

protegido legalmente como Paraje Natural desde 2003. Ello ha favorecido que su fauna terrestre sea relativamente bien conocida, con unas 140 especies citadas hasta la fecha (Paracuellos et al. 2006). A pesar de que los vertebrados identificados ascienden casi al centenar de especies, los quirópteros han sido tan desconocidos en la localidad, que la única cita existente hasta hace poco más de 10 años fue un registro puramente descriptivo de actividad de quirópteros no identificados, por Paracuellos et al. (2006). Los autores reportaron que la información disponible hasta entonces provenía en su totalidad del personal laboral allí afincado, el cual observó ejemplares "en vuelo y cazando en los alrededores del faro y sobre la llanura superior de la isla, respectivamente a finales de abril y mediados de septiembre de 2004", sin datos más precisos de identificación. No obstante, a finales de la década de 2000, el 19 de noviembre de 2009, Yus et al. (2013) describieron la presencia del primer ejemplar de murciélago en la isla, identificado como Tadarida teniotis. Desde entonces no se ha reportado ningún otro avistamiento. En la presente aportación, 10 años más tarde, presentamos oficialmente la segunda y tercera citas de quirópteros para la isla. Los días 27 y 28 de septiembre de 2019 se observa por segunda vez un individuo de la especie T. teniotis en la parte exterior del faro (Fig. 2A). Además, el mismo 27 de septiembre se captura un tercer ejemplar de murciélago, identificado como Hypsugo savii (Bonaparte, 1837) (Fig. 2B). Para la última especie ésta sería la primera cita en la isla de Alborán. Tales ejemplares acabaron por entrar incidentalmente a las dependencias militares del faro. Dadas sus dificultades para volver a salir espontáneamente a cielo abierto, se les capturó para su inmediata liberación al exterior. No fueron medidos datos biométricos de los
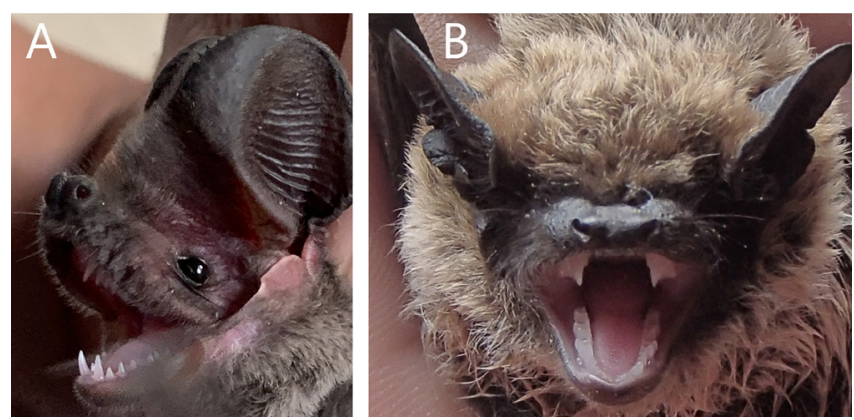

Fig. 2 - Ejemplares de A: Tadarida teniotis (Rafinesque, 1814) y B: Hypsugo savii (Bonaparte, 1837) capturados por el personal militar en el interior del faro de la isla de Alborán el 27 de septiembre de 2019 (foto: J. Tortolero).

murciélagos, de modo que su identificación se realizó exclusivamente con las imágenes tomadas durante la captura, apoyándose en Dietz \& von Helversen (2004) y el asesoramiento de expertos (C. Ibáñez). Las fotografías disponibles no permitieron determinar el sexo y la edad de los ejemplares. Sin embargo, el grado de osificación de las articulaciones alares que se aprecia en el $H$. savii induce a pensar que podría tratarse de un individuo nacido antes del 2019. Al margen de esos contactos, entre los días 28 y 30 de marzo de 2020 fueron avistados volando sobre la plataforma central de la isla hasta un máximo de cinco nuevos quirópteros que no pudieron llegar a identificarse.

El mar de Alborán se caracteriza por su escasez en islas, en la mayoría de los casos, además, contiguas a las costas vecinas. Dada su proximidad a los continentes, en algunas de ellas han llegado a documentarse nutridas comunidades 
de quirópteros (Jiménez-Robles et al. 2018). Sin embargo, colonias residentes de murciélagos parecen estar ausentes en la isla de Alborán a tenor de los resultados aquí expuestos. $Y$ es que, a pesar de lo frecuentado del lugar por el hombre dada su importancia estratégica y ecológica (Paracuellos et al. 2006), hasta la fecha no se tiene constancia más que de estas citas aisladas de murciélagos (2004, 2009, 2019 y 2020), sin existir estudios específicos ni indicios conocidos que puedan indicar una persistencia del grupo a más largo plazo en la isla. Ello viene apoyado por el hecho de que estas nuevas observaciones de murciélagos se han realizado en tan solo cinco días concretos, a pesar de los aproximadamente 300 días de estancia en la isla durante el período 2018-2020, en los que se han hecho allí observaciones regulares de fauna. Esto probablemente se debe al grado de aislamiento y al reducido tamaño del enclave, lo que podría dificultar su asentamiento por minimización de recursos disponibles, como alimento y agua dulce.

No obstante, a pesar de las posibles limitaciones para ser residentes, ambas especies citadas en la presente nota se encuentran ampliamente distribuidas por sendas costas cercanas de África y la península ibérica (RomeroZarco 1990, Wilson \& Reeder 2005). Además, T. teniotis tiene cierta capacidad de movimiento, siendo considerada parcialmente migratoria en ambas orillas continentales (Benda \& Piraccini 2016, Balmori 2017). Por su parte, $H$. savii es una especie capaz de migrar según algunos autores (Prieto 2002). Los mencionados datos sugieren que los recorridos de individuos desde las costas adyacentes a este remoto enclave marítimo pudieran ser abarcables por sendas especies. Tal información, en su conjunto, deriva en la hipótesis de que la presencia de quirópteros en la isla de Alborán probablemente pudiera no ser fruto más que de ejemplares esporádicos en movimientos dispersivos. No obstante, los mencionados hallazgos tienen importancia porque podrían estar poniendo de manifiesto que, a pesar de la gran lejanía existente entre los márgenes norte y sur del mar de Alborán a la altura de su solitaria isla central (con unos $150 \mathrm{~km}$ de distancia entre ambos), pudiera haber posibilidad de vuelos intercontinentales directos de quirópteros entre sus orillas meridionales y septentrionales. Estos datos ganan trascendencia teniendo en cuenta que, aunque ha sido documentado el desplazamiento de murciélagos a mar abierto en otras partes Europa (Amengual et al. 2007, Boshamer \& Bekker 2008, Ahlén et al. 2009, Rydell et al. 2014, Hüppop \& Hill 2016), podrían apoyar la constatación de la posible migración pre y post-reproductiva (dados los contactos siempre primaverales y otoñales constatados) de estas especies entre Europa y África (ya apuntado por Romero-Zarco 1990). En cualquier caso, de existir estos desplazamientos deberían estar principalmente dándose, por proximidad, a través de los tan solo $15 \mathrm{~km}$ existentes de costa a costa a la altura del estrecho de Gibraltar como sugieren García-Mudarra et al. (2009). En este sentido, un dato que pudiera apoyar ese contacto poblacional fluido entre las dos costas es que, en el caso de T. teniotis, no existe diferenciación genética entre las poblaciones de Marruecos y España (García-Mudarra et al. 2009, Amorim et al. 2020), lo cual es compatible con posibles desplazamientos frecuentes entre ambos continentes. Por otro lado, para $H$. savii las poblaciones ibéricas y africanas están bien diferenciadas biométrica y genéticamente a nivel mitocondrial, lo que estaría indicando aislamiento geográfico entre ambas poblaciones (Romero-Zarco 1990, García-Mudarra et al. 2009). No obstante, y pesar de ello, se ha encontrado algún caso esporádico de individuos con haplotipos africanos en la península Ibérica de dicha especie, lo que podría también ser reflejo de la existencia de algún desplazamiento ocasional entre ambas costas (García-Mudarra et al. 2009).

El estudio de la posible conectividad de las poblaciones al norte y al sur de Alborán debería ser, entonces, objeto de una mayor atención futura, con el fin de poder constatar más fehacientemente la entidad de los potenciales desplazamientos intercontinentales por parte de las diferentes especies de murciélagos.

\section{AGRADECIMIENTOS}

Se agradece a Carlos Ibáñez su asesoramiento para la identificación de los ejemplares, así como a Philip Kramer su revisión del inglés. Los comentarios aportados por los tres revisores anónimos y Jesús Benzal contribuyeron a una mejora sustancial del artículo.

\section{REFERENCIAS}

AHLÉN, I., BAAG $\varnothing E$, H. J. \& BACH, L. (2009). Behavior of Scandinavian bats during migration and foraging at sea. Journal of Mammalogy, 90(6): 1318-1323. https://doi. org/10.1644/09-MAMM-S-223R.1

AMENGUAL, B., LÓPEZ-ROIG, M. \& SERRA-COBO, J. (2007). First record of seasonal over sea migration of Miniopterus schreibersii and Myotis capaccinii between Balearic Islands (Spain). Acta Chiropterologica, 9(1): $\quad 319-322 . \quad$ https://doi.org/10.3161/17335329(2007)9[319:FROSOS]2.0.CO;2

AMORIM, F., RAZGOUR, O., MATA, V. A., LOPES, S., GODINHO, R., IBÁÑEZ, C., JUSTE, J., ROSSITER, S. J., BEJA, P. \& REBELO, H. (2020). Evolutionary history of the European free-tailed bat, a tropical affinity species spanning across the Mediterranean Basin. J Zool Syst Evol Res, 58(1): 499518. https://doi.org/10.1111/jzs.12326

BALMORI, A. (2017). Murciélago rabudo - Tadarida teniotis (Rafinesque, 1814). En: Enciclopedia Virtual de los Vertebrados Españoles. ed.: Museo Nacional de Ciencias Naturales (Consejo Superior de Investigaciones Científicas). Madrid, España.

BAROVA, S. \& STREIT, A. (2018). Action plan for the conservation of all bat species in the European Union 2018-2024. ed.: European Commision \& UNEP/Eurobats. Brussels, Belgium, 86 pp.

BENDA, P. \& PIRACCINI, R. (2016). Tadarida teniotis. The IUCN Red List of Threatened Species 2016: e.T21311A22114995. https://doi.org/10.2305/IUCN. UK.2016-2.RLTS.T21311A22114995.en 
BISSON, I. -A., SAFI, K. \& HOLLAND, R. A. (2009). Evidence for repeated independent evolution of migration in the largest family of bats. PLOS ONE, 4(10): e7504. https:// doi.org/10.1371/journal.pone.0007504

BOSHAMER, J. P. C. \& BEKKER, J. P. (2008). Nathusius' pipistrelles (Pipistrellus nathusii) and other species of bats on offshore platforms in the Dutch sector of the North Sea. Lutra, 51(1): 17-36.

DIETZ, C. \& VON HELVERSEN, O. (2004). Illustrated Identification key to the bats of Europe. Version 1.0. Electronic Publication. ed.: Dietz, C. \& von Helversen, O. Tuebingen \& Erlangen, Germany, 72 pp.

GARCÍA-MUDARRA, J. L., IBÁÑEZ, C. \& JUSTE, J. (2009). The Straits of Gibraltar: barrier or bridge to Ibero-Moroccan bat diversity?. Biological Journal of the Linnean Society, 96(2): 434-450. https://doi.org/10.1111/j.10958312.2008.01128.x

HEDENSTRÖM, A. (2009). Optimal migration strategies in bats. Journal of Mammalogy, 90(6): 1298-1309. https:// doi.org/10.1644/09-MAMM-S-075R2.1

HÜPPOP, O. \& HILL, R. (2016). Migration phenology and behaviour of bats at a research platform in the southeastern North Sea. Lutra, 59(1-2): 5-22.

HUTTERER, R., IVANOVA, T., MEYER-CORDS, C. \& RODRIGUES, L. (2005). Bat migration in Europe. A review of banding data and literature. ed.: Federal Agency for Nature Conservation. Bonn, Germany, 180 pp.

IBÁÑEZ, C. (2007). Myotis punicus (Felten, Spitzenberger y Storch, 1977). En: Atlas y Libro Rojo de los Mamíferos Terrestres de España. ed.: Dirección General para la Biodiversidad-SECEM-SECEMU. Madrid, España, p.163165.
JIMÉNEZ-ROBLES, O., TENA, E., MARTÍN, J. \& DE PAZ, O. $(2018,7$ y 8 Diciembre). Primeros datos de los murciélagos de las islas Chafarinas, norte de África: un estudio preliminar con acústica. VII Jornadas SECEMU, Gibraltar 7 y 8 Diciembre 2018.

PARACUELLOS, M., NEVADO, J. C. \& MOTA, J. F. (2006). Entre África y Europa. Historia natural de la Isla de Alborán. ed.: RENPA \& Consejería de Medio Ambiente (Junta de Andalucía). Sevilla, España, 265 pp.

POPA-LISSEANU, A. G. \& VOIGT, C. C. (2009). Bats on the move. Journal of Mammalogy, 90(6): 1283-1289. https:// doi.org/10.1644/09-MAMM-S-130R2.1

PRIETO, S. G. (2002). Hypsugo savii (Bonaparte, 1837). En: Atlas de los Mamíferos Terrestres de España. ed.: Dirección General para la Biodiversidad-SECEM-SECEMU. Madrid, España, 190-193.

ROMERO-ZARCO, P. (1990). Quirópteros de Andalucía y Marruecos. Universidad de Sevilla. Sevilla, España, 737 pp.

RYDELL, J., BACH, L., BACH, P., DIAZ, L. G., FURMANKIEWICZ, J., HAGNER-WAHLSTEN, N., KYHERÖINEN, E. -M., LILLEY, T., MASING, M., MEYER, M. M. et al. (2014). Phenology of migratory bat activity across the Baltic Sea and the south-eastern North Sea. Acta Chiropterologica, 16(1): 139-147. https://doi.org/10.3161/150811014X683354

WILSON, D. E. \& REEDER, D. M. (2005). Mammal species of the world: a taxonomic and geographic reference. 3a ed. ed.: Johns Hopkins University Press. Baltimore, Maryland, United States of America, 2142 pp.

YUS, R., PARACUELLOS, M., GONZÁLEZ, J. A., GARCÍA, H., TORRES, M. A., BUENO, I., GÁMEZ, S., JEREZ, D. \& CABO, J. M. (2013). IV. La Isla de Alborán. En: Historia natural de la región de Melilla (Guelaya, Alborán y Chafarinas). ed.: Fundación Gaselec. Melilla, España, 151 pp. 